\title{
REFLECTANCE RECONSTRUCTION OF HYPERSPECTRAL IMAGE BASED ON GAUSSIAN SURFACE FITTING
}

\author{
Shuqiang Lyu ${ }^{1,2}$, Chunhao Huang ${ }^{1}$, Miaole Hou ${ }^{1,2} *$ \\ 1 School of Geomatics and Urban Spatial Informatics, Beijing University of Civil Engineering and Architecture, Beijing 100044, \\ China-(lvshuqiang, houmiaole)@bucea.edu.cn, 2108160218004@stu.bucea.edu.cn \\ 2 Beijing Key Laboratory for Architectural Heritage Fine Reconstruction \& Health Monitoring, Beijing 102616, China.
}

\section{Commission III, WG III/4}

KEY WORDS: Hyperspectral imaging, Reflectance, Reconstruction, Calibration, Gaussian, Surface fitting

\begin{abstract}
:
Different from the field of remote sensing, artificial lights are often utilized as the energy source for spectral imaging in the ground hyperspectral applications. The kind of double-spot light source is widely adopted in some large scale ground hyperspectral applications. However, it is hard to reach a satisfied lighting without difference in light intensity in many cases although the lamps are tuned carefully. Therefore, a reflectance calibration of hyperspectral imaging based on the data of diffuse reflectance standard and Gaussian surface fitting is proposed in this paper. The purpose is to improve the reconstruction accuracy of hyperspectral reflectance image by minimized the error caused by the uneven illumination of artificial light source. The method has a higher accuracy than traditional one according to the experiment results.
\end{abstract}

\section{INTRODUCTION}

Hyperspectral imaging technology has the advantages of high spectral resolution and ability to obtain both spectral and spatial information, which is one of the most significant revolution since the emergence of remote sensing technology (Qingxi, 2016). With the development of hyperspectral remote sensing technology, it has been widely applied to medical imaging and processing, agricultural yield estimation, food safety testing, environmental monitoring and other fields (Bing, 2016). Reflectance is one of the natural attributes of ground objects, which is the basic data for hyperspectral remote sensing. Therefore, it is necessary to transform the radiance data storage in digital number $(\mathrm{DN})$ value of the image into reflectance before further quantitative studies such as material identification, endmember extraction, abundance calculation, and so on.

The kind of reflectance calibration method based on Diffuse Reflectance Standard (DRS) is commonly used in hyperspectral imaging. In recent years, many improved method have been proposed in some works. Geladi, P., et al. (Geladi, 2004) as well as James B. and Paul G. (2006) suggested that the calibration method can be improved by using several DRS with different reflectance values $(99 \%, 75 \%, 50 \%, 25 \%$ or $2 \%)$. In order to deal with the reflectance calibration problem of the hyperspectral image for apples, the data of a DRS with reflectance of $75 \%$ were adopted as the dark current data to improve the accuracy of the reflectance calibration (Xing, 2005). Another modification was carried out (Gómez-Sanchis et al., 2008), where a geometric correction factor in particular for spherical objects like citrus was utilized. Diwan P. Ariana and Renfu Lu (2008) and Noviyanto et al. (Noviyanto, 2019) proposed the idea of a dynamic DRS, which considered that an image would be under different lighting conditions in different spatial positions. Therefore, the combination of transmittance and reflectance was combined to improve accuracy.

Those works can improve the accuracy of the reflectance calibration method based on DRS to some extent. However, most size of the objects mentioned above are small and they can be measured in a light room that can provide an ideal environment with uniform light intensity distribution. As for the application in remote sensing, the electromagnetic wave is come from the Sun and the ground can be regarded as approximate Lambertian target. They are observed under the assumption that they are illuminated evenly.

In the actual cultural heritage protection and restoration project, there are many large immovable cultural heritage such as temple fresco. It is necessary to use artificial light sources to perform hyperspectral imaging on a large scale, where it is hard to create an ideal environment with uniform light intensity distribution. Therefore, for these kinds of applications, this paper proposes an improved reflectance calibration method based on surface fitting, which can solve the problem of reflectance calibration in the case of uneven light distribution caused by artificial light sources in large indoor scenes. And it is verified by hyperspectral images acquired in laboratory.

\section{METHODOLOGY}

\subsection{Artificial light source}

Different from the field of remote sensing, artificial lights are often used as the energy source for spectral imaging in the ground hyperspectral applications such as cultural heritage protection, industrial testing, and food safety. The light source generally chooses halogen lamps, xenon lamps, etc., which still have good radiant energy in the short-wave infrared band. According to the spatial distribution, light sources can be divided into double-spot light sources, multi-spot light sources, and linear light sources. According to the actual purpose of working applications, all three kinds are widely adopted. In this paper, we mainly study the double-spot light source.

Double-spot light source is that two lights are generally fixed on the left and right sides of the hyperspectral imager in a fixed spatial position respectively. The spatial distribution of imager

\footnotetext{
* Corresponding author. houmiaole@bucea.edu.cn (Miaole Hou).
} 
and lights is shown in Figure 1. Such kind of light source has the advantages of simple installation and convenient operation. And it is also efficient in data acquisition because it is no need to adjust the light source and camera parameters every time when the hyperspectral imager moved along its linear slide rail parallel to the relative large scale target.

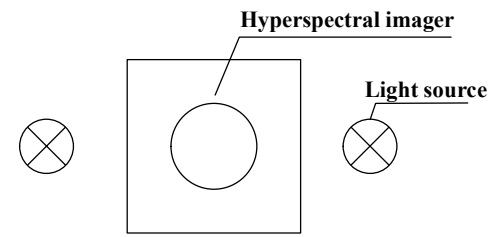

Figure 1. The position of the light source and imager

Although the angle and the focusing adjustment of lamps have been tuned elaborately, it is hard to reach a satisfied lighting without difference in light intensity in many cases. In order to quantitatively evaluate the spatial distribution of the light intensity of the double-spot light source, two bands are selected at random from the images collected from a DRS with known $99 \%$ reflectance for analysis. The distributions of their DN value are shown in Figure 2, where the $\mathrm{x}$-axis and $\mathrm{y}$-axis are the spatial coordinate system of imaging with pixel as the unit, and the DN value are plotted on z-axis. We can find that the $\mathrm{DN}$ value is high in the centre and low in the edge, and gradually declines from the center to the edge. It shows that the light intensity distribution of double-spot light source is uneven.

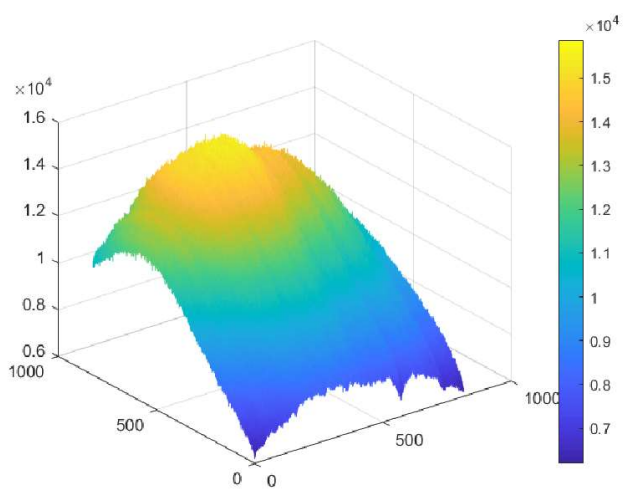

(a) DN value of $440^{\text {th }}$ band $(644 \mathrm{~nm})$

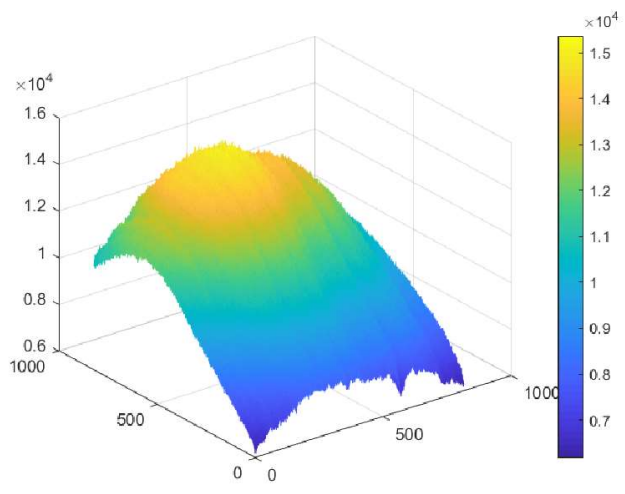

(b) $\mathrm{DN}$ value of $509^{\text {th }}$ band $(687 \mathrm{~nm})$

Figure 2. DN distribution of DRS data

At the same time, we use the mean value, standard deviation and Coefficient of Variation (CV) to evaluate the DN value distribution of bands concerned. The results is shown in table 1 .

\begin{tabular}{|c|c|c|c|}
\hline Band & Mean & $\begin{array}{c}\text { Standard } \\
\text { Deviation }\end{array}$ & CV \\
\hline $440^{\text {th }}$ & 12419.378 & 4061.603 & 0.327 \\
$509^{\text {th }}$ & 12241.758 & 3872.045 & 0.316 \\
\hline
\end{tabular}

Table 1. Evaluate Results of DN

When the standard deviation is bigger, the data dispersion is bigger. Similarly, the bigger the $\mathrm{CV}$ is, the bigger the data dispersion is. From the Table 1, we can find that the intensity distribution of double-spot light source is uneven and the variation degree is high. Therefore, it is necessary to consider this factor in reflectance correction.

\subsection{Reflectance calibration based on DRS}

The traditional reflectance calibration method based on DRS utilizes formula (1) to calculate the reflectance.

$$
\boldsymbol{R}=\frac{\boldsymbol{R}_{\text {raw }}-\boldsymbol{R}_{\text {dark }}}{\boldsymbol{R}_{\text {white }}-\boldsymbol{R}_{\text {dark }}}
$$

where $\quad \boldsymbol{R}_{\text {raw }}=$ original hyperspectral image data collected

$\boldsymbol{R}_{\text {white }}=$ the image data collected with DRS

$\boldsymbol{R}_{\text {dark }}=$ the dark current data

$\boldsymbol{R}=$ the reflectance corrected

In order to ensure the accuracy of reflectance reconstruction, multiple DRSs are frequently used for correction. Therefore, equation (1) can be written as:

$$
\boldsymbol{R}=\boldsymbol{R}_{\text {raw }} \cdot \frac{1}{\boldsymbol{R}_{\text {white }}-\boldsymbol{R}_{\text {dark }}}+\left(-\frac{\boldsymbol{R}_{\text {dark }}}{\boldsymbol{R}_{\text {white }}-\boldsymbol{R}_{\text {dark }}}\right)
$$

After simplification, it would become:

$$
R=R_{\text {raw }} \cdot \boldsymbol{a}+\boldsymbol{b}
$$

Generally, several reference plates with different known reflectance are used. From the centre position of each reference plate, a small area with the same size and location is resampled to average its DN value as $\boldsymbol{R}_{\text {raw }}$ in formula (3) during the calibration process. The average value of dark current is calculated as $\boldsymbol{R}_{\text {dark. }}$. And the coefficient $\boldsymbol{a}$ and $\boldsymbol{b}$ can be figured out by the least square method using the data measured multiple times on multiple reference plates.

\subsection{Reflectance calibration by Gaussian surface fitting}

Considering on the problem of uneven light intensity distribution, this paper proposes a new method to reconstruct the reflectance image, which takes into account the nonuniformity of light intensity distribution and the regularity of its change. The method uses the data based on DRS to fit the Gaussian surface best, so as to minimize the root mean square error between the Gaussian surface and data of DRS. The flow chart is shown in Figure 3. The Gaussian surface calculated will be used as the standard reference data to calibrate the hyperspectral image point by point to obtain the reflectance data. The specific steps are as follows:

Step 1. To determine whether the acquired DRS data is full of the actual shooting area or not. If yes, then go to step 4 directly, and take the DRS data as $\boldsymbol{R}_{\boldsymbol{w h}}$ ite .

Step 2. To calculate the parameters of Gaussian surface. From the hyperspectral image with the DRS, the largest rectangular area within the coverage of the DRS is resampled to fit the 
Gaussian surface. The parameters of the surface model are calculated by the least square method.

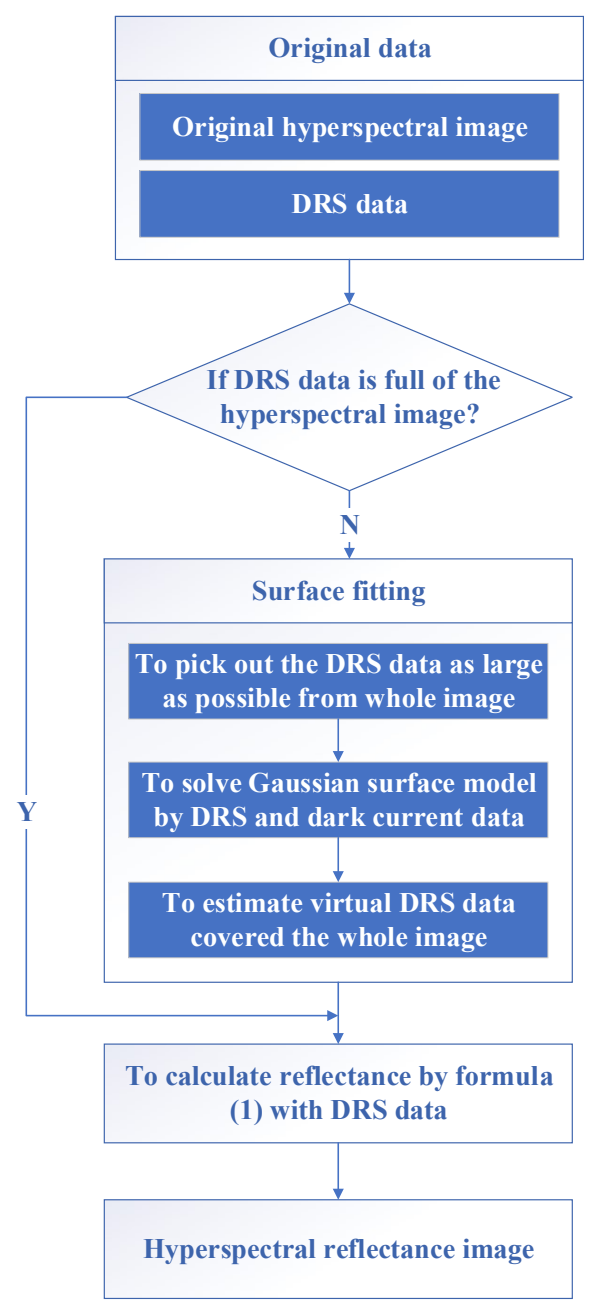

Figure 3 Flow chart of Gaussian surface fitting calibration

Step 3. The surface model is used to estimate a virtual DRS data as $\boldsymbol{R}_{\text {white }}$ that will cover the whole hyperspectral image.

Step 4. The reflectance of hyperspectral image is corrected by using formula (1), point by point and band by band.

Among them, the key is the selection of the surface model. Different surface models directly affect the subsequent prediction results.

For most of the laser beams, the ideal light intensity distribution should meet the Gaussian distribution (Chen, 2015). Through the analysis of the light intensity distribution in hyperspectral imaging, a light intensity model of double-spot light source on the DRS is introduced as formula (4).

$$
I(x, y)=A \cdot \exp \left\{-\left[\frac{\left(x-x_{o}\right)^{2}}{2 \sigma_{1}^{2}}+\frac{\left(y-y_{o}\right)^{2}}{2 \sigma_{2}^{2}}\right]\right\}
$$

Where $I(x, y)=$ the light intensity at the point $(x, y)$, DN value $A=$ the maximum value of the light intensity in the DRS $\left(x_{0}, y_{0}\right)=$ the central position with the light intensity of $A$ $\delta_{1}, \delta_{2}=$ the standard deviation in two directions

\section{EXPERIMENT AND RESULT}

The instrument used in this paper is VNIR400H ground hyperspectral imager with $2.8 \mathrm{~nm}$ spectral resolution covering $400-1000 \mathrm{~nm}$ wavelength. The spatial resolution is $1392 \times 1000$ pixels. The DRS used is produced by Labsphere Company, with reflectivity of $99 \%$. The light source used is a halogen lamp produced by USHIO Company of Japan. The light source is fixed in the way of double-spot light source.

The test object is a red cotton cloth fixed on a flat board, covering the whole scanning range of a hyperspectral scene. In an ideal case, the reflectance obtained should be the same in whole image because of the same substance to be measured. Therefore, we calibrated the same hyperspectral image by the method based on Gaussian surface fitting and traditional one to compare their performance. The results of reflectance calibrated from one single band is given in Figure 4 as an example. For the purpose of calculating and displaying convenience, the data plotted in the Figure 4 is the result of the reflectance multiplied by 100 .

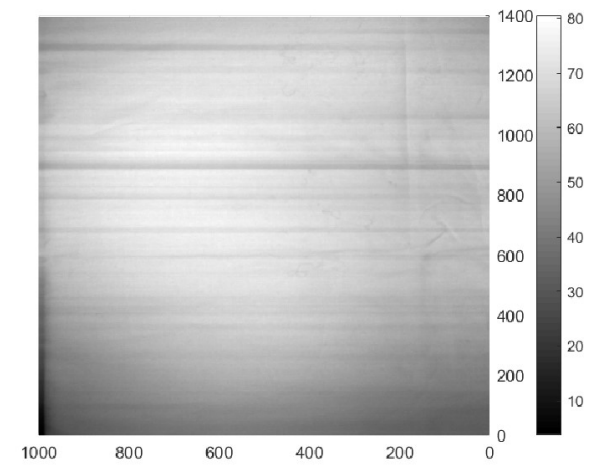

(a) Result by Gaussian surface fitting method

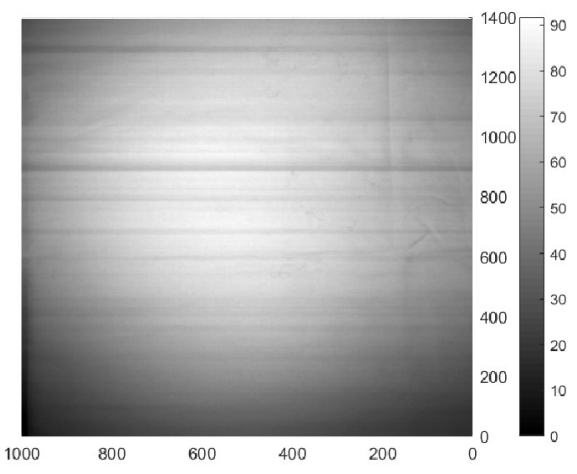

(b) Result by traditional method

Figure 4 . The results of two methods of $440^{\text {th }}$ band $(644 \mathrm{~nm})$

In order to evaluate the reconstruction accuracy quantitatively, the mean, standard deviation and the coefficient of variation of the whole bands are calculated and plotted in Figure 5. 


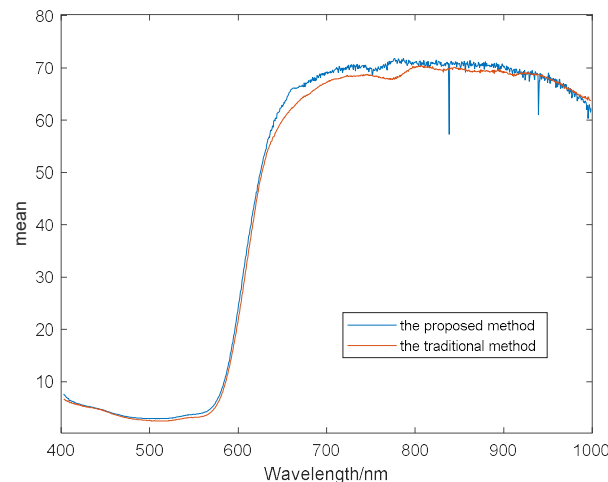

(a) Mean

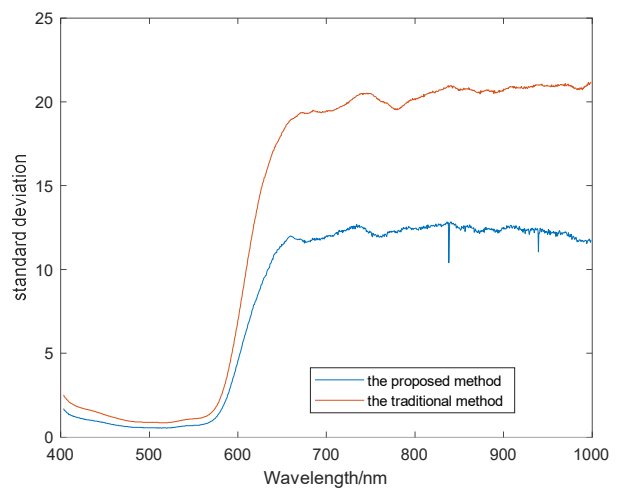

(b) Standard deviation

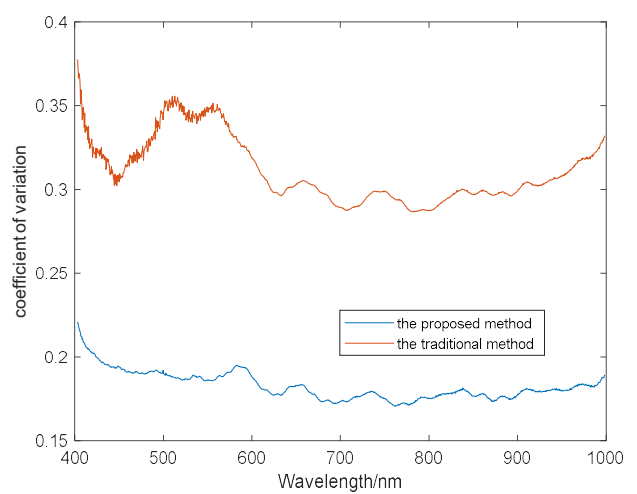

(c) Coefficient of variation

Figure 5. The statistics result of hyperspectral image

Because the experimental object is a homogeneous material, the reflectance calibrated should be a constant for a single band theoretically. In fact, due to various noises and other factors of the image, it should be a random number that fluctuates around its mean value for a single band. Therefore, the smaller the standard deviation and CV value, the better the reflectance correction effect in this experiment. It can be seen from the Figure 5 that the standard deviation and the $\mathrm{CV}$ of the method proposed in this paper are both smaller than those of the traditional method. This phenomenon shows that the data distribution is more even after using the method proposed in this paper. Therefore, the accuracy of this method is higher than that of the traditional method.

In order to show the results more intuitively, we randomly selected a band of data to calculate its histogram. It is $440^{\text {th }}$ band with wavelength of $644 \mathrm{~nm}$. The results are shown in Figure 6.

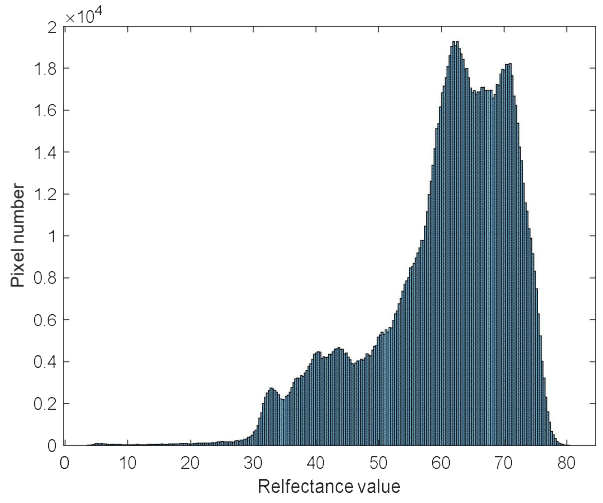

(a) By proposed method

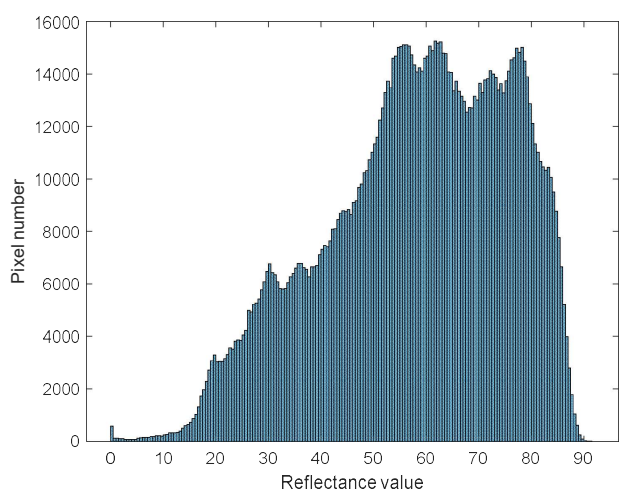

(b) By traditional method

Figure 6 . The histogram of $440^{\text {th }}$ band $(644 \mathrm{~nm})$

From the histogram in Figure 6, it can be found that the result of the proposed method is more concentrated, which means it has a higher calibration accuracy for evenly distributed data in this experiment.

\section{CONCLUSIONS}

An experiment was carried out to study the light intensity distribution of double spot light used in the ground hyperspectral imaging in this paper. It was found that the light intensity is high in the centre and low in the edge, and it declines from the centre to the four sides. Therefore, the influence of light source must be taken into account in the ground hyperspectral reflectance reconstruction.

This paper proposed a reflectance reconstruction method based on Gaussian surface fitting. We used the data collected with DRS and known reflectance to fit a Gaussian surface model by the least square method to estimate a virtual DRS covered the whole hyperspectral images. The reflectance of the hyperspectral image is calibrated by the virtual DRS and dark current data. The calibration results showed that the method proposed in this paper can reduce the influence of uneven light intensity to a large extent, and the accuracy of reconstruction is higher than that by the traditional one in all band.

On the other hand, it also be noticed that the method proposed in this paper is time-consuming for a higher computational complexity, because it is necessary to perform primary surface fitting for each band. Further study should be carried out to reach a higher accuracy as well as efficiency. 


\section{ACKNOWLEDGEMENTS}

This research was funded by the Research Fund of the National Key Research and Development Program (No. 2017YFB1402105), and Beijing Advanced Innovation Center for Future Urban Design (No. UDC2016030200), and Great Wall Scholars Training Program Project of Beijing Municipality Universities (No. CIT\&TCD20180322)

\section{REFERENCES}

Binbin C., Qiang Z., Yaodong L., et al., 2015. Fast attenuation algorithm of laser intensity based on three-dimensional Gaussian fitting. High Power Laser and Particle Beams, 27(04):10-14

Bing Z., 2016. Advancement of hyperspectral image processing and information extraction, Journal of remote sensing, 20(05):1062-1090.

Diwan P. Ariana, Renfu Lu., 2008. Quality evaluation of pickling cucumbers using hyperspectral reflectance and transmittance imaging: Part I. Development of a prototype. Sensing \& Instrumentation for Food Quality \& Safety, 2(3):144151.

Geladi, P., Burger, J., Lestander, T., 2004, Hyperspectral imaging: calibration problems and solutions. Chemometrics and Intelligent Laboratory Systems, 72(2):209-217.

Gómez-Sanchis J., Moltó E., Camps-Valls G., et al., 2008. Automatic correction of the effects of the light source on spherical objects. An application to the analysis of hyperspectral images of citrus fruits. Food Eng, 85: 191-200.

James B., Paul G., 2006. Hyperspectral NIR image regression part I: calibration and correction. Journal of Chemometrics, 19(5-7):355-363.

Noviyanto, A., Abdulla, W., 2019. Segmentation and calibration of hyperspectral imaging for honey analysis. Computers and Electronics in Agriculture, 159:129-139.

Qingxi T., Bing Z., Lifu Z., 2016. Current progress of hyperspectral remote sensing in China. Journal of Remote Sensing, 20(05): 689-707.

Xing J., 2005. Detecting bruises on 'golden delicious' apples using hyperspectral imaging with multiple wavebands. Biosystems Engineering, 90(1):27-36. 